\title{
Genetic regulators of leaf size in Brassica crops
}

\author{
Umer Karamat ${ }^{1}$, Xiaoxue Sun (1) ${ }^{1}, \mathrm{Na} \mathrm{Li}^{1}$ and Jianjun Zhao ${ }^{1}$
}

\begin{abstract}
Leaf size influences plant development and biomass and is also an important agricultural trait in Brassica crops, in which leaves are the main organ produced for consumption. Leaf size is determined by the coordinated regulation of cell proliferation and cell expansion during leaf development, and these processes are strictly controlled by various integrated signals from the intrinsic regulatory network and the growth environment. Understanding the molecular mechanism of leaf size control is a prerequisite for molecular breeding for crop improvement purposes. Although research on leaf size control is just beginning in Brassica, recent studies have identified several genes and QTLs that are important in leaf size regulation. These genes have been proposed to influence leaf growth through different pathways and mechanisms, including phytohormone biosynthesis and signaling, transcription regulation, small RNAs, and others. In this review, we summarize the current findings regarding the genetic regulators of leaf size in Brassica and discuss future prospects for this research.
\end{abstract}

\section{Introduction}

Brassica is one of the most economically important genera of Brassicaceae. It includes a variety of horticultural crops, such as cabbage, rape, mustard, and cauliflower. Brassica crops are a vital source of vegetables, cooking oil, and condiments for human consumption ${ }^{1,2}$. Moreover, Brassica plants are rich in genetic diversity and phenotypic variation, providing abundant genetic resources for crop breeding and ideal materials for plant development studies ${ }^{3}$.

As the most important organ for photosynthesis, leaves produce the main source of energy not only for plant growth but also for human nutrition and other purposes $^{4,5}$. The size and shape of leaves affect light energy utilization, thereby influencing plant development and biomass. In addition, leaves are critical for many physiological processes, such as photorespiration, transpiration, and temperature regulation; therefore, leaf size can also impact plant fitness and stress responses ${ }^{6}$. In Brassica vegetables, whose leaves are the main organ of

Correspondence: Na Li (13521251421@163.com) or

Jianjun Zhao (jjz1971@aliyun.com)

'State Key Laboratory of North China Crop Improvement and Regulation, Key Laboratory of Vegetable Germplasm Innovation and Utilization of Hebei, Collaborative Innovation Center of Vegetable Industry in Hebei, College of Horticulture, Hebei Agricultural University, 071000 Baoding, China consumption, the leaves are a nutrient source, providing vitamins, soluble fiber, manganese, and glucosinolates ${ }^{7}$. The size of the leaves of these crops is a major factor affecting plant size and yield. On the other hand, the ideal leaf size and plant size are of great importance to the appearance of these vegetables. Thus, leaf size is a key agricultural trait for these crops.

Although leaf development and growth are affected by various environmental signals, leaf size is intrinsically controlled by complex genetic networks that regulate cell proliferation and cell expansion during leaf development. After leaf primordia are initiated at the edge of the shoot apical meristem (SAM), leaves grow and reach their mature size through cell proliferation and cell expansion. In the early stage, most cells in the primordia divide continuously, resulting in a rapid increase in the cell number; in contrast, the cell size remains relatively constant ${ }^{8}$. Subsequently, a transition occurs in which most cell division ceases, and cell expansion predominates, thereby leading to cell enlargement ${ }^{8}$. Thus, the size of mature leaves is determined by both the cell number and the cell size ${ }^{9-11}$.

The genetic mechanisms underlying leaf size control have been extensively studied in the model plant Arabidopsis thaliana (A. thaliana), which is also a member of the Brassicaceae family. Through forward and reverse 
genetic studies, numerous genes involved in leaf size regulation have been identified ${ }^{8,12}$. These genes function in different signaling pathways or regulatory modules to influence cell proliferation and cell expansion. As there is a wide range of genomic collinearity between $A$. thaliana and Brassica plants ${ }^{3,13}$, studies in A. thaliana are especially helpful for understanding the mechanisms of leaf size control in Brassica plants and for identifying Brassica leaf size regulators by homolog identification. With the rapid progression of molecular studies in Brassica plants and the availability of genomic sequences for several Brassica crops, studies on leaf size control in Brassica crops are increasing; several genes and quantitative trait loci (QTLs) controlling leaf growth have been identified and characterized in Chinese cabbage (B. rapa L. ssp. pekinensis), cauliflower (B. oleracea L. var. botrytis), turnip (B. rapa L. ssp. rapa), and rapeseed (B. napus). In this review, we will discuss the leaf size regulators identified so far and highlight their possible roles in leaf growth.

\section{Leaf size regulators involved in phytohormone homeostasis or signaling}

Phytohormones play crucial roles in plant development and responses to biotic and abiotic stresses. Recent studies have demonstrated the functions of auxin, ethylene, abscisic acid (ABA), and gibberellic acid (GA) in Brassica leaf growth. Several genes involved in the homeostasis of or signaling by these phytohormones have been identified as leaf size regulators in both $A$. thaliana and Brassica crops.

\section{Regulators involved in auxin signaling}

Auxin biosynthesis, transport, and signaling are essential for plant growth. Auxin participates in plant development and growth by regulating cell division, cell growth, and cell differentiation. Several auxin-related genes have been found to play vital roles in maintaining the final leaf size in Brassica crops.

\section{BrANT}

AINTEGUMENTA (ANT) encodes an APETALA 2/ ETHYLENE RESPONSE FACTOR (AP2/ERF) family transcription factor that responds to auxin and controls downstream gene expression to regulate organogenesis and cell proliferation ${ }^{14,15}$. In $A$. thaliana, ANT overexpression resulted in large leaves due to increased cell proliferation, indicating that $A N T$ promotes leaf growth by regulating cell division ${ }^{16}$. In Chinese cabbage, three $A N T$ and six ANT-LIKE (BrAIL) genes have been identified, and the expression of the BrANT genes and three of the BrAIL genes was responsive to auxin treatment ${ }^{17}$. Among these homologs, BrANT-1 shows the highest protein sequence similarity to the $A$. thaliana ANT protein (AtANT). The overexpression of the Chinese cabbage BrANT-1 gene in A. thaliana increased leaf size due to enhanced cell proliferation, indicating that the effect of BrANT-1 on leaf growth is similar to that of AtANT. Equally important, $A$. thaliana plants overexpressing BrANT-1 also exhibited a significantly increased stomata number and a slightly increased net photosynthetic rate. The expression of STOMAGEN was also significantly enhanced in the transgenic plants. Thus, BrANT-1 may also regulate stomatal density by upregulating the $S T O$ MAGEN gene ${ }^{17}$.

\section{BrARGOS}

AUXIN-REGULATED GENE INVOLVED IN ORGAN SIZE (ARGOS) is believed to function downstream of auxin and upstream of $A N T$ to regulate cell proliferation and organ growth ${ }^{18}$. ARGOS encodes a protein of low molecular weight with an ORGAN SIZE RELATED (OSR) domain ${ }^{19}$. ARGOS-overexpressing A. thaliana plants had large leaves, while antisense $A R G O S$ plants had smaller leaves than the wild-type plants ${ }^{18}$. Furthermore, ARGOS can regulate the expression of $A N T$, and the large-leaf phenotype of $A R G O S$-overexpressing plants depends on the functional $A N T$, indicating that $A N T$ acts downstream of $A R G O S$ to regulate organ growth ${ }^{18}$. The overexpression of the Chinese cabbage BrARGOS gene in A. thaliana plants increased the size of leaves and other organs due to enhanced cell proliferation ${ }^{20}$. Furthermore, semiquantitative RT-PCR analysis detected increased $A N T$ expression in transgenic plants, revealing a conserved mechanism of BrARGOS for leaf size control similar to that of AtARGOS. Interestingly, a study on a polyploidy Chinese cabbage series revealed that the expression level of ARGOS was concomitantly upregulated with the increase in the ploidy level and the size of the leaves and petals, indicating that the expression of $A R G O S$ is regulated by genome size ${ }^{21}$. However, the mechanistic basis of this regulation remains unclear.

\section{pPLAIIIS}

The patatin-related phospholipase A (pPLA) family member PLAIII $\delta$ influences organ size by regulating auxin distribution. The pPLA family enzymes hydrolyze membrane glycerolipids into lysoglycerolipids and free fatty acids and play vital roles in hormone biosynthesis and signaling, as well as other biological processes ${ }^{22}$. In $A$. thaliana, ten pPLAs are divided into three subfamilies, namely, pPLAI, pPLAII $(\alpha, \beta, \gamma, \delta, \varepsilon)$, and pPLAIII $(\alpha, \beta, \gamma$, $\delta)^{23}$. The overexpression of $p$ PLAIII $\delta$ in A. thaliana or $B$. napus resulted in shorter leaves, floral organs, and siliques but thicker stems than those of the wild type, indicating that pPLAIII $\delta$ inhibits longitudinal growth but promotes transverse expansion during organ growth ${ }^{24}$. Furthermore, transgenic plants showed increased phosphatidic acid (PA) concentrations and free IAA levels in aboveground organs, revealing that pPLAIII $\delta$ may regulate 
auxin distribution by regulating PA. Interestingly, the pavement cells of transgenic leaves produced fewer indentations and lobes than those of the wild type, resulting in a relatively simple network of epidermal cells and indicating that pPLAIII $\delta$ is also involved in polar cell growth. However, the role of Brassica pPLAs in leaf growth has not yet been reported.

\section{BrARP1 and BrDRM1}

AUXIN-REPRESSED PROTEIN 1 (ARP1) and DORMANCY-ASSOCIATED PROTEIN 1 (DRM1) encode auxin-repressed small-peptide proteins that are conserved among several plant species. These genes have been reported to be highly expressed in nongrowing tissues and dormant buds, where they are associated with dormancy $^{25}$. For example, BrARP1 and BrDRM1 from Chinese cabbage have been identified as negative regulators of plant growth. The overexpression of BrARP1 or BrDRM1 in A. thaliana plants reduced root, hypocotyl, and leaf growth due to suppressed cell elongation or cell expansion $^{26}$. As these genes are upregulated in plants exposed to abiotic stress, it is likely that they inhibit growth, allowing plants to overcome stress by redirecting their resources.

\section{Regulators functioning in ethylene signaling}

Ethylene is an endogenously synthesized hormone that exists in gas form. It is involved in many developmental processes and stress responses, such as dormancy, seed germination, leaf expansion, disease and pest attack, and tissue damage. It is also essential in agriculture, where it functions in organ senescence, abscission, and fruit ripening ${ }^{27}$. In Brassica crops, two important components of the ethylene signaling pathway have been recognized as leaf size regulators.

\section{BrERF4}

ETHYLENE RESPONSE FACTORS (ERFs) encode AP2/ ERF superfamily transcription factors that are central components of the ethylene signaling pathway by regulating the transcription of ethylene-responsive genes ${ }^{28}$. In A. thaliana plants, AtERF4 has been reported to affect many developmental and stress response processes, such as leaf senescence, anthocyanin synthesis, the plant defense response, and the iron deficiency response ${ }^{29-32}$. During iron deficiency, an erf4 mutant exhibited enhanced chlorophyll content and plant growth, while the transient overexpression of AtERF4 in Nicotiana tabacum leaves promoted chlorophyll degradation ${ }^{32}$. Similarly, overexpressing the Chinese cabbage BrERF4 in $A$. thaliana plants reduced leaf size by inhibiting cell expansion $^{33,34}$. The expression of two EXPANSIN (EXP) genes, AtEX1A5 and AtEX1A10, was downregulated in BrERF4-overexpressing A. thaliana plants, revealing that
BrERF4 restricts cell expansion by repressing EXP genes $^{34}$. However, it seems that BrERF4 regulates the expression of these genes indirectly, as no ERF binding site was identified in the promoter regions of AtEX1A5 and AtEX1A10. In addition, it has been demonstrated that the ERF4 gene from Chinese cabbage and other plant species is also involved in stress tolerance and disease resistance ${ }^{33,35,36}$. It will be of great importance to study how ERF4 mediates stress-induced growth retardation by reducing cell expansion.

\section{BoCDAG1}

CURD DEVELOPMENT ASSOCIATED GENE 1 (CDAG1) encodes a protein with an OSR domain. It was identified as a growth stimulator in cauliflower due to its high expression in young cauliflower curds ${ }^{19}$. The overexpression of CDAG1 in A. thaliana plants increased the size of leaves, flowers, roots, and other organs. Similarly, the ectopic expression of CDAG1 in cauliflower promoted plant growth and increased plant biomass. Therefore, CDAG1 could be an ideal target for the genetic engineering of high-yield crops. Although CDAG1 is homologous to A. thaliana ARGOS and ARGOSE-LIKE (ARL), which also contain an OSR domain, these three proteins seem to play different roles in organ growth regulation. Specifically, ARGOS promotes organ growth by enhancing cell proliferation ${ }^{18}$, while ARL mainly influences cell expansion; ${ }^{37}$ by contrast, CDAG1-overexpressing plants showed increased cell number and cell size ${ }^{19}$. A promoting effect on both cell proliferation and cell expansion was also reported for another OSR family gene, namely, $O S R 1^{38}$, in A. thaliana. Unexpectedly, the overexpression of CDAG1 upregulates several ERF genes, whereas BrERF4 overexpression inhibits cell expansion and leaf growth $^{33,34}$. Further studies are needed to clarify the mechanism of CDAG1 in growth regulation.

\section{Regulators involved in abscisic acid (ABA) and gibberellic acid (GA) signaling BnNCED3}

$\mathrm{ABA}$ is a phytohormone associated with various stress responses. It regulates many developmental and physiological processes, such as osmosis, seed development, seed germination, leaf senescence, stomatal closure, and bud dormancy. Although defects in vegetable growth have been reported in several ABA-deficient mutants, ABA is generally considered to be a growth inhibitor under stress conditions. For example, it restricts leaf growth by repressing vegetative leaf emergence, promoting flowering, and accelerating leaf senescence ${ }^{39}$. Activating AtNCED3, which encodes a key enzyme of ABA biosynthesis, enhanced ABA biosynthesis, restricted plant growth, and enhanced drought tolerance ${ }^{40-42}$. The ortholog of AtNCED3 from B. napus, BnNCED3, has 
been demonstrated to play a similar role in repressing plant growth. The overexpression of BnNCED3 in $A$. thaliana plants increased ABA biosynthesis and enhanced abiotic stress tolerance and leaf senescence, indicating the importance of $\mathrm{ABA}$ in integrating environmental cues and developmental signals to regulate leaf senescence ${ }^{43}$.

\section{BrRGA1}

GA is a plant hormone that functions in various growth and developmental processes, including leaf expansion, stem elongation, flowering transition, and seed germination ${ }^{44}$. GA-deficient and GA-insensitive mutants generally show a dwarf phenotype and have been extensively used in plant breeding to improve lodging resistance. The famous "Green Revolution" gene in wheat encodes a central repressor of GA signaling named DELLA ${ }^{45}$. DELLAs are plant-specific GRAS family transcription factors that repress GA-responsive genes. GA perception leads to the degradation of DELLA proteins and, therefore, the activation of downstream genes ${ }^{44,46}$. The widely utilized modern semidwarf wheat cultivars carry the Reduced height-1 allele that encodes a mutant form of the DELLA protein, which causes GA insensitivity and decreased stem elongation ${ }^{47}$. DELLA proteins are highly conserved among dicotyledon and monocotyledon species, and different paralogs play distinct but somewhat overlapping roles in the GA response ${ }^{44,46}$. The A. thaliana genome encodes five DELLA proteins. Mutations leading to DELLA stabilization resulted in dwarf phenotypes, whereas the loss of function of four of the DELLA proteins promoted leaf growth through both cell division and cell expansion ${ }^{48,49}$. In B. rapa, a semidominant dwarf mutant, $d w f 2$, was insensitive to exogenous GA3 ${ }^{50}$. Comparative mapping analysis showed that DWF2 encodes BrRGA1 (Bra024875), which is homologous to the A. thaliana DELLA protein RGA (repressor of ga1-3). The mutant Brrga1-d protein has an amino acid substitution in a conserved motif required for protein degradation, thereby leading to the accumulation of the protein $^{51}$. Although DELLA proteins were shown to restrain leaf growth in $A$. thaliana, the Brrga1-d mutant has not been reported to affect leaf size. It is unclear whether other DELLA proteins in $B$. rapa function redundantly with $B r R G A 1$ to regulate leaf growth.

\section{Control of leaf growth in Brassica through the integration of hormone signaling}

Based on the current findings, we propose a possible model of leaf size control through integrated hormone signaling (Fig. 1). First, auxin promotes leaf growth by inducing BrARGOS and BrANT to accelerate cell proliferation and repressing $B r A R P 1$ and $B r D R M 1$ to enhance cell expansion ${ }^{17,20,26}$. Meanwhile, ethylene restricts cell expansion and leaf growth through BrERF4

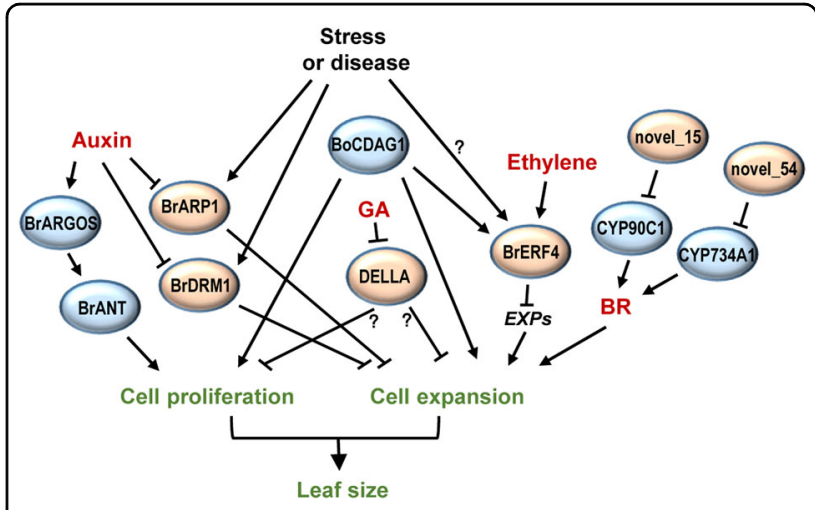

Fig. 1 Control of leaf size in Brassica through the integration of hormone signaling. Leaf size is influenced by auxin, ethylene and brassinosteroids (BRs). The role of GA in leaf size control in Brassica remains unclear. The positive and negative regulators are shown in blue and yellow ovals, respectively. Arrows indicate positive regulation, and T indicates negative regulation. Question marks indicate undefined regulation

by downregulating EXP genes ${ }^{33,34}$. Although the expression of some $E R F$ genes is induced by BoCDAG1, BrERF4 and BoCDAG1 have the opposite effect on leaf size ${ }^{19,33,34}$. Therefore, it remains unclear whether BoCDAG1 functions through ethylene signaling to control leaf growth. In addition, brassinosteroids (BRs) promote cell expansion and leaf growth" ${ }^{52}$ (as discussed in "MicroRNAs that regulate leaf size"). In Arabidopsis, GA positively regulates leaf growth through both cell proliferation and cell expansion. The loss of function of the GA signalingrepressing DELLA proteins leads to enlarged leaf size ${ }^{48,49}$. In $B$. rapa, the mutation of the DELLA protein BrRGA1 causes a dwarf phenotype; ${ }^{50,51}$ however, the role of Brassica DELLA proteins in leaf size control remains poorly understood.

Notably, several negative regulators of leaf size have been shown to respond to stress or disease. For instance, the expression of BrARP1 and BrDRM1 can be induced by abiotic stresses ${ }^{26}$, and the ERF4 genes from Chinese cabbage and other plant species are also involved in stress tolerance and disease resistance ${ }^{33,35,36}$. It is likely that under stress or disease conditions, these genes can mediate signals to restrict leaf growth. Furthermore, increasing ABA biosynthesis through the overexpression of $B n N C E D 3$ can enhance abiotic stress tolerance and leaf senescence ${ }^{43}$, indicating that $\mathrm{ABA}$ can accelerate leaf senescence under abiotic stress.

\section{Other transcription factors that regulate leaf size}

Transcription factors contain a specific DNA binding domain and a transcriptional regulation region. They bind to the cis-regulatory elements of their target genes to repress or activate gene transcription. Transcription factors play key roles in many developmental and 
physiological processes by controlling gene expression. In addition to the aforementioned transcription factors, several other transcription factors have been found to regulate leaf growth.

\section{BrrTCP2}

Members of the TCP family of plant-specific transcription factors contain a 59-amino acid basic helix-loop-helix (bHLH) motif defined as the TCP domain, which is named after TEOSINTE BRANCHED1 (TB1) from maize (Zea mays), CYCLOIDEA (CYC) from Antirrhinum majus, and PROLIFERATING CELL FACTORS (PCFs) from rice (Oryza sativa) ${ }^{53}$. Numerous TCP genes have been identified in various plant species, and TCP proteins are grouped into two classes according to the subtle differences in their TCP domains. Class I TCPs have been proposed to promote plant growth, while Class II TCPs have been reported to prevent cell proliferation and plant growth ${ }^{53}$. The $A$. thaliana genome encodes 24 TCP proteins. TCP2, TCP3, TCP4, TCP10, and TCP24 are class II TCPs that function redundantly in leaf growth. The loss of function of these genes resulted in enlarged leaves and wrinkled leaf margins due to excessive cell division. In turnip, 39 TCPs have been identified ${ }^{54}$. The overexpression of BrrTCP2 rescued the phenotype of the $A$. thaliana tcp2/4/10 triple mutant, and the overexpression of BrrTCP2 in wild-type A. thaliana plants reduced the cell number and leaf size, indicating that BrTCP2 limits leaf growth by inhibiting cell proliferation $^{54}$. In addition, miR319a-targeted BrpTCP4 has been reported to regulate the size and shape of heads of Chinese cabbage (refer to the MicroRNAs section for details).

\section{BrGRFs}

Members of the GROWTH-REGULATING FACTOR (GRF) family of small, plant-specific transcription factors function in growth and development ${ }^{55,56}$. GRFs form protein complexes with the transcriptional coactivators GRF-INTERACTING FACTORS (GIFs) ${ }^{55,56}$. In A thaliana, nine GRF and three GIF genes have been identified. Although most GRFs have been found to positively regulate leaf size, they seem to play different roles in leaf growth. For example, AtGRF1-3 function redundantly to enhance cell expansion ${ }^{57}$, while AtGRF4 functions in cell proliferation in leaves and modulates the development of the SAM and cotyledons; ${ }^{58}$ by contrast, AtGRF5 interacts with GIF1/ANGUSTIFOLIA3 (AN3) to enhance cell proliferation in leaf primordia ${ }^{59}$. Genome-wide analysis identified 17 GRFs in Chinese cabbage ${ }^{60}$. The overexpression of BrGRF3-1/3-2/5/7/8-1/8-2/9 in A. thaliana plants increased the size of cotyledons, leaves, flowers, siliques, and seeds, as well as the seed oil content. Furthermore, the enhanced organ growth of the transgenic plants resulted from increased cell proliferation but not cell expansion, indicating that these BrGRFs stimulate organ growth by promoting cell proliferation ${ }^{61}$. In $A$. thaliana plants, GRF1-4 and GRF7-9 are targeted by miR396, and the expression of miR396 is regulated by $\mathrm{TCP}^{62,63}$. However, it is unclear whether this regulatory module exists in Brassica species.

\section{BrNGA1}

NGATHA (NGA) proteins belong to the plant-specific B3 superfamily ${ }^{64}$. NGA1-4 from $A$. thaliana form a small protein subgroup and play key roles in leaf and flower development. The quadruple mutant (nga1 nga2 nga3 nga4) produces wide and serrated leaves and abnormal flowers, while the overexpression of these genes reduces leaf growth ${ }^{65,66}$. The B. rapa genome encodes four NGA1, three NGA2, two NGA3, and two NGA4 proteins. The overexpression of BrNGA1 in A. thaliana resulted in small and narrow leaves. Kinematic analyses confirmed that BrNGA1 affected both the rate and the duration of cell proliferation in the leaves. Consistently, low levels of $C y c B 1 ; 1$ and $C y c D 3 ; 1$ were observed in leaves overexpressing $B r N G A 1$, suggesting that $B r N G A 1$ regulates organ growth by limiting cell proliferation ${ }^{67}$. In $A$. thaliana plants, NGA-like proteins (NGALs) have been shown to restrict organ growth by repressing (KLUH) KLU expression $^{68}$. The role of KLU in organ growth has not been reported in Brassica species.

\section{MicroRNAs that regulate leaf size}

MicroRNAs (miRNAs) are endogenous RNA sequences of $\sim 22$ nucleotides that are key components of gene regulatory networks in plants and animals ${ }^{69}$. Pre-miRNAs are transcribed from the corresponding miRNA genes and processed by several endonucleases to yield mature miRNAs that are subsequently incorporated into the RNA-induced silencing complex (RISC). miRNAs mediate the targeting of RISC to the corresponding mRNAs and promote the degradation of target mRNAs ${ }^{70}$. In $A$. thaliana plants, several miRNAs have been reported to play key roles in regulating leaf growth ${ }^{63,71,72}$. Some evidence suggests that miRNAs are also important leaf size regulators in Brassica crops.

\section{MiR319}

MiR319 is a key regulator of leaf development that targets $T C P$ genes ${ }^{71,72}$. In the $A$. thaliana jaw-D mutant, the increased expression of miR319 downregulated TCPs, resulting in large and highly wrinkled leaves. In Chinese cabbage, miR319a has been demonstrated to regulate the size and shape of the cabbage heads ${ }^{73}$. Furthermore, the overexpression of BrpMIR319a2 in Chinese cabbage altered the expression pattern of BrpTCP4, leading to the excess growth of both apical and interveinal regions and resulting in enlarged and cylindrical heads. 


\section{MiR394}

In $A$. thaliana plants, miR394 and its target gene $L E A F$ CURLING RESPONSIVENESS (AtLCR) are essential regulators of leaf morphology and stem cell maintenance ${ }^{74,75}$. The lcr mutant and miR394-overexpressing plants were semidwarf with large and upward curling leaves. In contrast, the overexpression of miR394-resistant $L C R$ resulted in the downward curling of leaves ${ }^{74}$. In rapeseed, the overexpression of $m i R 394$ downregulated $B n L C R$, delayed flowering time and increased the size of leaves, pods, and seeds ${ }^{76}$. In addition, transgenic rapeseed overexpressing the antisense mRNA of $B n L C R$ produces large leaves, and that overexpressing $B n L C R$ exhibits decreased leaf size. These studies revealed that miRNA394 and $B n L C R$ play conserved functions in regulating leaf growth in rapeseed $^{76}$. It is unclear whether $B n L C R$ regulates cell proliferation or cell expansion processes in leaf growth.

\section{Novel_15 and novel_54}

In Chinese cabbage, small leaf and plant sizes were observed in autotetraploid plants $(4 \mathrm{X})$ created from a doubled haploid (2X) line. Transcriptome analysis showed that two miRNAs, novel_15 and novel_54, were upregulated and that their target genes (BraA01000252 and BraA05004386, encoding CYP90C1 and CYP734A1, respectively), which are both involved in BR biosynthesis, were downregulated. Consistently, the autotetraploid plants showed low BR levels compared with the haploid line, indicating that these miRNAs may be involved in leaf growth through their regulation of BR biosynthesis ${ }^{52}$.

\section{MiRNA regulatory network for leaf size control}

The function of miRNAs and their targets in leaf size control has been extensively studied in the model plant $A$. thaliana. The miR319-TCPs-miR396-GRFs module plays central roles in regulating leaf growth. In this module, class II TCP transcription factors restrict cell proliferation during leaf growth, while GRFs promote cell division. TCPs and GRFs are targeted by miR319 and miR396, respectively. Meanwhile, TCPs can regulate the expression of miR396 to modulate the transcription of GRFs. Some evidence suggests that this regulatory module is likely conserved in Brassica (Fig. 2). In Chinese cabbage, miR319a-targeted BrpTCP4 has been found to regulate the size of cabbage heads, and BrrTCP2 from turnip was also identified as a negative regulator of leaf size. However, it remains unknown whether BrrTCP2 is targeted by miR319 and whether Brassica TCPs can regulate the expression of miR396. Another miRNA, miR394, targets LCR to regulate leaf growth in rapeseed and is similar to that in A. thaliana. Moreover, two novel miRNAs, novel_15 and novel_54, were identified as negative leaf size regulators targeting two genes involved in BR biosynthesis ${ }^{52}$. In combination, miRNAs and

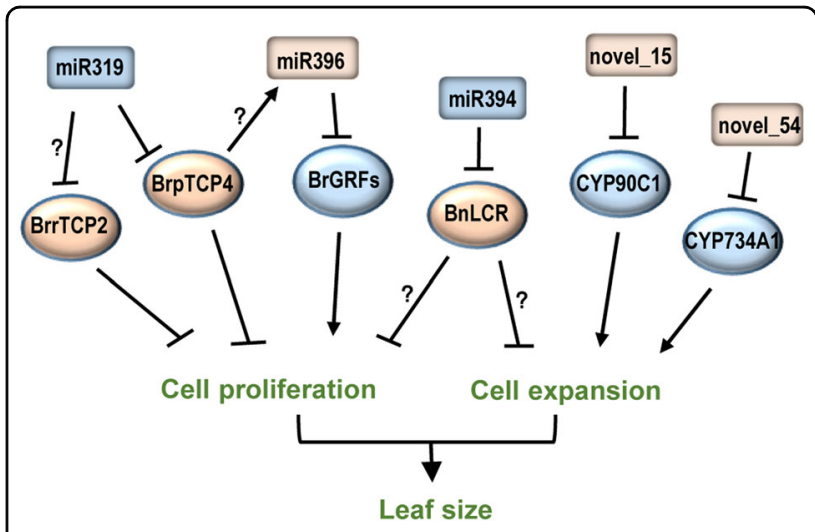

Fig. 2 MiRNAs and their targets involved in Brassica leaf size regulation. MiRNAs are presented in rectangles, and their targets are presented in ovals. The positive and negative regulators are shown in blue and yellow, respectively. Arrows indicate positive regulation, and $T$ indicates negative regulation. Question marks indicate undefined regulation

their targets are key regulators controlling leaf growth in Brassica (Fig. 2).

\section{Regulators involved in other pathways BnDA1}

$D A 1$ encodes a ubiquitin-dependent protease that negatively regulates organ size ${ }^{77,78}$. The $A$. thaliana da1-1 mutant produced large leaves, flowers, and seeds. The da1-1 allele encodes a mutant protein with an arginineto-lysine substitution at position 358 (DA1 $\left.{ }^{\mathrm{R} 358 \mathrm{~K}}\right)$. This mutant protein has a negative effect on DA1 and its homolog, DA1-related (DAR1), in A. thaliana by prolonging the duration of cell proliferation and increasing the size of organs. The overexpression of $D A 1^{R 358 K}$ in wild-type $A$. thaliana plants increased organ size, which mimicked the phenotype of plants with a simultaneous disruption of $D A 1$ and $D A R 1^{77}$. In contrast, the overexpression of wild-type $D A 1$ inhibited plant growth ${ }^{79}$. The BnDA1 protein from $B$. napus is highly homologous to $A$. thaliana DA1, with $83.15 \%$ amino acid sequence similarity. The overexpression of $B n D A 1$ restored the phenotype of the $A$. thaliana da1-1 mutant, and $B$. napus plants overexpressing $A t D A 1^{R 358 K}$ showed increased seed, cotyledon, leaf, flower, and silique size as well as increased biomass. Furthermore, candidate gene association analyses suggested that $B n D A 1$ also contributes to seed weight ${ }^{80}$. These studies demonstrated that DA1 has a conserved function related to organ size control in Brassica species and can be used for biomass improvement. In A. thaliana plants, the protease activity of DA1 can be activated by ubiquitination, and active DA1 can cleave the TCP family transcription factors TCP14/15 to regulate organ growth ${ }^{78,81}$. It would be worthwhile to 
investigate whether BrDA1 acts through BrTCP transcription factors to regulate leaf size in Brassica species.

\section{FCA-RRM}

FLOWERING CONTROL LOCUS A (FCA) is a key regulator of floral transition ${ }^{82}$. It regulates the expression of the floral repressor FLOWERING LOCUS C (FLC) by affecting the alternative polyadenylation of $F L C$ antisense transcripts $^{83,84}$. The FCA protein contains two RNA-binding domains (RRMs) that are highly conserved among different plant species ${ }^{85}$. Intriguingly, these RRM domains have been reported to promote plant growth. The overexpression of FCA-RRM1 and FCA-RRM2 can increase cell size in rice and cotton, thereby improving the yield ${ }^{85-87}$. Similarly, $B$. napus plants overexpressing B. napus FCA-RRM2 exhibited increased cell size and organ size. Notably, the expression of the cyclin-B2-1 gene was remarkably downregulated in transgenic rapes overexpressing FCA-RRM2. However, whether the increase in cell size is caused by the decreased expression of cyclin-B2-1 remains elusive $^{88}$.

\section{BrPHYB}

Phytochrome B (PHYB) is a red-light receptor that mediates light signaling and plant development. It is involved in various developmental processes, such as seed germination, organ growth, flowering, and the shadeavoidance response ${ }^{89}$. The loss of function of PHYB in $A$. thaliana and other plants led to constitutive symptoms of shade-avoidance syndrome (SAS), such as longer internodes, taller plants, and lower tiller numbers ${ }^{90-92}$. In contrast, the overexpression of $P H Y B$ inhibited hypocotyl elongation $^{93}$. A recent study demonstrated that ectopic expression of the Chinese cabbage $P H Y B$ gene in $A$. thaliana plants decreased leaf length and plant height. Surprisingly, AtPHYB-overexpressing plants flowered earlier than wild-type plants, whereas the overexpression of $B r P H Y B$ in A. thaliana caused late flowering under shortday conditions. Further analyses showed that $B r P H Y B$ likely delayed flowering by repressing the expression of gibberellin biosynthesis genes, while AtPHYB promoted flowering by upregulating FLOWERING LOCUS T; these results indicate that $B r P H Y B$ and $A t P H Y B$ have conserved functions with regard to cell elongation but divergent roles with regard to flowering time ${ }^{94}$.

\section{QTLs of leaf size control}

QTLs are genomic regions that influence the inheritance of quantitative traits. In recent years, QTL mapping has been widely used to identify candidate genes for leaf traits in different crops, such as tomato, maize, grape, and A. thaliana ${ }^{95-98}$. In Brassica, a number of QTLs of leaf traits have been identified using different mapping populations. Some of these QTLs colocalized with genes whose homologs in A. thaliana have been identified as key regulators of leaf development and growth. These studies provided useful information for further identification of leaf size regulators in Brassica.

Using F2 populations derived from crosses of rapidcycling Brassica to three B. oleracea varieties, 47 QTLs influencing plant size were detected by restriction fragment length polymorphisms (RFLPs). The QTLs related to lamina length were found to correspond to five ancestral genes, namely, REVOLUTA (REV), AUXIN RESISTANT 1 (AXR1), AXR3, AXR4, and ASYMMETRIC LEAVES 2 (AS2), which are essential for leaf initiation and morphogenesis in A. thaliana plants ${ }^{99}$.

In B. rapa, three QTLs for plant height and ten QTLs for leaf traits were detected by analyzing three different types of populations developed from wide crosses between $B$. rapa accessions ${ }^{100}$. Similarly, three QTLs for leaf blade length and three QTLs for leaf blade width were identified by using different populations constructed from two Chinese cabbage inbred lines, 'Chiifu402-42' and 'Kenshin-40-43'; these QTLs coincided with CYCB2;4, CYCD3;1, ULT1, AN3, and ANT, which are involved in cell cycle and leaf growth regulation ${ }^{101}$. By using a doubled haploid population derived from a cross between the oil type cultivar yellow sarson (ssp. trilocularis) and the vegetable type cultivar pak choi (ssp. chinensis), 167 QTLs for leaf traits were detected. Several QTLs for leaf size colocalized with the key regulators of leaf development in A. thaliana, including BrGRF5_A01, ASYMMETRIC LEAVES1 (BrAS1)_A03, BrFLC5_A03, LONGIFOLIA1 (BrLNG1)_A10, PINHEAD (BrPNH) _A09, SQUAMOSA PROMOTER BINDING PROTEINLIKE5 (BrSPL5)_A01, and BrSPL5_A05. Transcript analysis showed that the expression of BrLNG1_A10 was positively correlated with leaf size. Genetic regulatory network analysis revealed that $B r C y c B 2 ; 4 \_A 07, B r A R$ GOS_A07, BrARL_A03, and BrCYCB1_A01 were also associated with leaf size and plant architecture ${ }^{102}$.

In B. napus, 31 QTLs related to leaf morphology traits have been identified using recombinant inbred lines (RILs) constructed from the parental lines GH06 and P174. For leaf size regulation, eight QTLs for petiole length, two QTLs for lamina width, five QTLs for lamina length, eight QTLs for lamina size ratio, and two QTLs for total leaf size were detected. Gene expression analyses revealed that several regulators of leaf shape and size were differentially expressed in lines with distinct leaf shapes, including AS2, gibberellin 20-oxidase 3 (GA20OX3), gibberellin-regulated family genes, GRF, and KNOTTED1-like homeobox genes $(K N A T S)^{103}$.

\section{Conclusion and future prospects}

The size of a mature leaf is regulated by two partially overlapping processes, namely, cell proliferation and cell expansion. For Brassica crops, especially for leafy vegetables, leaf size influences not only plant biomass and crop 


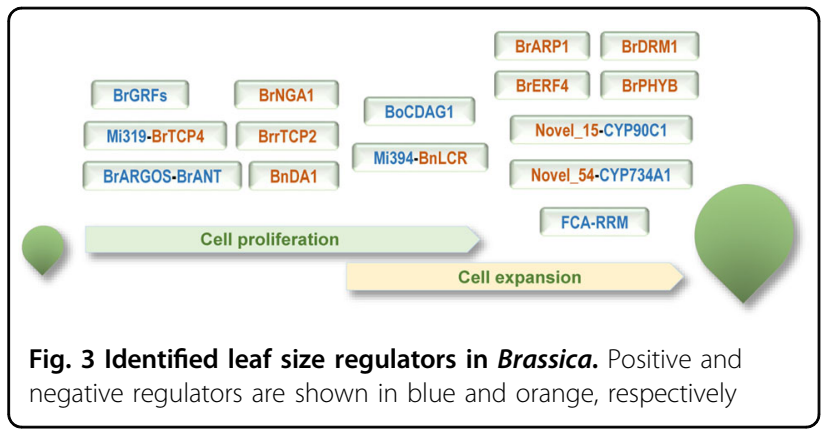

yield but also plant size and appearance quality. Although recent studies have identified several genetic regulators of leaf size in Brassica species, our knowledge of leaf size control in Brassica crops is still poor. In A. thaliana plants, nearly a hundred genes have been found to influence leaf size; ${ }^{8,12}$ by contrast, only a small portion of the corresponding Brassica homologs have been characterized (Fig. 3, Table 1), implying that a large number of leaf size regulators have yet to be discovered. On the other hand, a large proportion of the Brassica leaf size regulators reported so far have been identified through homologous gene identification. Due to the unavailability of transgenic technology, A. thaliana transgenic lines were used to verify gene function in most cases. Although there is evidence that the leaf size control functions of these genes are largely conserved, the effects of the loss of function or overexpression of these genes in Brassica plants are uncertain. Compared with A. thaliana, Brassica plants have experienced a whole-genome triplication (WGT) event, so that numerous $A$. thaliana genes have multiple copies in Brassica species. Furthermore, it was reported that phytohormone-responsive genes were significantly overretained in the $B$. rapa and $B$. oleracea genomes $^{3}$. The complexity of Brassica genomes may have contributed to the abundant morphological diversity of Brassica plants and may also have created more complicated and species-specific genetic networks for leaf size regulation. Therefore, there is an urgent need to investigate the role of the identified regulators of leaf development in different Brassica crops.

The development of whole-genome sequencing technology and the MutMap method has enabled the identification of novel regulators by genome-wide association studies or mutant analyses. Currently, these studies are beginning to emerge in Brassica crops and are expected to make rapid progress at the forefront of functional genomics. In addition, the establishment of efficient genetic transformation methods and the use of geneediting technology will help to further verify the function of these regulators. The next challenge is to clarify the functionally redundant and divergent roles of the multiple copies of regulators and the interactions between

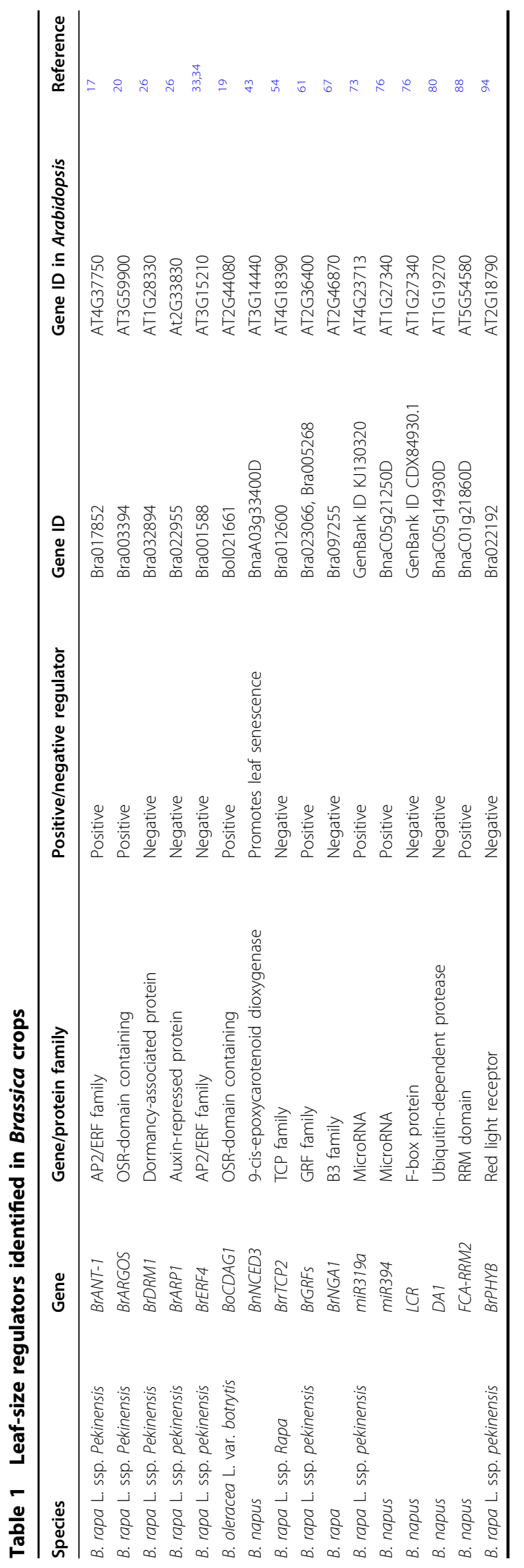


different regulators and finally to integrate the regulatory networks in different Brassica species. With the development of molecular breeding technologies, it has become possible to modulate the agricultural traits of crops by selecting or manipulating target genes. Identifying leaf size regulators in Brassica crops and understanding their function will inform the improvement of Brassica crops through the breeding high-yield crops with optimal leaf sizes as well as novel vegetable varieties with ideal leaf and plant sizes.

\section{Acknowledgements}

The authors thank our colleagues for their helpful suggestions for this review. We apologize to our colleagues whose work could not be discussed due to space limitations. Our research is supported by the National Key Research and Development Project (2016YFD0100204-17), the Natural Science Foundation of Hebei (C2020204111) and the Starting Grant from Hebei Agricultural University, China (YJ201920). We thank A \& L Scientific Editing (www.alpublish.com) for its linguistic assistance during the preparation of this manuscript.

\section{Author contributions}

U.K., X.S., N.L., and J.Z. wrote the review.

\section{Conflict of interest}

The authors declare no competing interests.

Received: 22 August 2020 Revised: 3 January 2021 Accepted: 24 February 2021

Published online: 01 May 2021

\section{References}

1. McAlvay, A. C. Domestication, Invasion, and Ethnobotany of Brassicarapa (The University of Wisconsin-Madison, 2018).

2. Rakow, G. Species origin and Economic Importance of Brassica in Brassica 3-11 (Springer, 2004).

3. Cheng, F., Wu, J., Liang, J. \& Wang, X. in The Brassica Rapa Genome (eds Wang, X. \& Kole, C.) 115-120 (Springer, 2015).

4. Zhu, X.-G., Long, S. P. \& Ort, D. R. Improving photosynthetic efficiency for greater yield. Annu. Rev. Plant Biol. 61, 235-261 (2010).

5. Edgerton, M. D. Increasing crop productivity to meet global needs for feed, food, and fuel. Plant Physiol. 149, 7-13 (2009).

6. Oguchi, R., Onoda, Y., Terashima, I. \& Tholen, D. in The Leaf: a Platform for Performing Photosynthesis (eds Adams III, W. W. \& Terashima, I.) 97-139 (Springer, 2018).

7. Nugrahedi, P. Y., Verkerk, R., Widianarko, B. \& Dekker, M. A mechanistic perspective on process-induced changes in glucosinolate content in Brassica vegetables: a review. Crit. Rev. Food Sci. Nutr. 55, 823-838 (2015).

8. Gonzalez, N., Vanhaeren, H. \& Inzé, D. Leaf size control: complex coordination of cell division and expansion. Trends Plant Sci. 17, 332-340 (2012).

9. Efroni, I., Eshed, Y. \& Lifschitz, E. Morphogenesis of simple and compound leaves: a critical review. Plant Cell 22, 1019-1032 (2010).

10. Breuninger, H. \& Lenhard, M. in Current Topics in Developmental Biology (ed. Wassarman, P. M.) Vol. 91, 185-220 (Elsevier, 2010).

11. Donnelly, P. M., Bonetta, D., Tsukaya, H., Dengler, R. E. \& Dengler, N. G. Cell cycling and cell enlargement in developing leaves of Arabidopsis. Dev. Biol. 215, 407-419 (1999).

12. Vercruysse, J., Baekelandt, A., Gonzalez, N. \& Inzé, D. Molecular networks regulating the cell division during leaf growth in Arabidopsis. J. Exp. Bot. 71, 2365-2378 (2019).

13. Cheng, F. et al. Subgenome parallel selection is associated with morphotype diversification and convergent crop domestication in Brassica rapa and Brassica oleracea. Nat. Genet. 48, 1218 (2016).

14. Elliott, R. C. et al. AINTEGUMENTA, an APETALA2-like gene of Arabidopsis with pleiotropic roles in ovule development and floral organ growth. Plant Cell 8, 155-168 (1996).
15. Klucher, K. M., Chow, H., Reiser, L. \& Fischer, R. L. The AINTEGUMENTA gene of Arabidopsis required for ovule and female gametophyte development is related to the floral homeotic gene APETALA2. Plant Cell 8, 137-153 (1996).

16. Nole-Wilson, S., Azhakanandam, S. \& Franks, R. G. Polar auxin transport together with AINTEGUMENTA and REVOLUTA coordinate early Arabidopsis gynoecium development. Dev. Biol. 346, 181-195 (2010).

17. Ding, Q. et al. Ectopic expression of a Brassica rapa AINTEGUMENTA gene (BrANT-1) increases organ size and stomatal density in Arabidopsis. Sci. Rep. 8, 1-13 (2018).

18. Hu, Y., Xie, Q. \& Chua, N.-H. The Arabidopsis auxin-inducible gene ARGOS controls lateral organ size. Plant Cell 15, 1951-1961 (2003).

19. $\mathrm{Li}, \mathrm{H}$. et al. Curd development associated gene (CDAG1) in cauliflower (Brassica oleracea L. var. botrytis) could result in enlarged organ size and increased biomass. Plant Sci. 254, 82-94 (2017).

20. Wang, B., Zhou, X., Xu, F. \& Gao, J. Ectopic expression of a Chinese cabbage BrARGOS gene in Arabidopsis increases organ size. Transgenic Res. 19, 461-472 (2010).

21. Gu, A. X. et al. Analyses of phenotype and ARGOS and ASY1 expression in a ploidy Chinese cabbage series derived from one haploid. Breed. Sci. 66, 161-168 (2016).

22. Scherer, G. F., Ryu, S. B., Wang, X., Matos, A. R. \& Heitz, T. Patatin-related phospholipase A: nomenclature, subfamilies and functions in plants. Trends Plant Sci. 15, 693-700 (2010).

23. Holk, A., Rietz, S., Zahn, M., Quader, H. \& Scherer, G. F. Molecular identification of cytosolic, patatin-related phospholipases A from Arabidopsis with potential functions in plant signal transduction. Plant Physiol. 130, 90-101 (2002).

24. Zheng, Y., Li, M. \& Wang, X. Proteomic insight into reduced cell elongation resulting from overexpression of patatin-related phospholipase PPLAll $\delta$ in Arabidopsis thaliana. Plant Signal. Behav. 9, e28519 (2014).

25. Rae, G. M., Uversky, V. N., David, K. \& Wood, M. DRM1 and DRM2 expression regulation: potential role of splice variants in response to stress and environmental factors in Arabidopsis. Mol. Genet. Genomics 289, 317-332 (2014).

26. Lee, J., Han, C.-T. \& Hur, Y. Molecular characterization of the Brassica rapa auxin-repressed, superfamily genes, BrARP1 and BrDRM1. Mol. Biol. Rep. 40, 197-209 (2013).

27. Ju, C. \& Chang, C. Mechanistic insights in ethylene perception and signal transduction. Plant Physiol. 169, 85-95 (2015).

28. Müller, M. \& Munné-Bosch, S. Ethylene response factors: a key regulatory hub in hormone and stress signaling. Plant Physiol. 169, 32-41 (2015).

29. Xu, X., Chen, C., Fan, B. \& Chen, Z. Physical and functional interactions between pathogen-induced Arabidopsis WRKY18, WRKY40, and WRKY60 transcription factors. Plant Cell 18, 1310-1326 (2006).

30. Koyama, T. \& Sato, F. The function of ETHYLENE RESPONSE FACTOR genes in the light-induced anthocyanin production of Arabidopsis thaliana leaves. Plant Biotechnol. 35, 87-91 (2018).

31. Koyama, T. et al. A regulatory cascade involving class II ETHYLENE RESPONSE FACTOR transcriptional repressors operates in the progression of leaf senescence. Plant Physiol. 162, 991-1005 (2013).

32. Liu, W. et al. The ethylene response factor AtERF4 negatively regulates the iron deficiency response in Arabidopsis thaliana. PLOS ONE 12, e0186580 (2017).

33. Seo, Y. J. et al. Overexpression of the ethylene-responsive factor gene BrERF4 from Brassica rapa increases tolerance to salt and drought in Arabidopsis plants. Mol. Cells 30, 271-277 (2010).

34. Park, J.-B. et al. Overexpression of stress-related genes, BrERF4 and AtMYB44, in Arabidopsis thaliana alters cell expansion but not cell proliferation during leaf growth. J. Plant Biol. 55, 406-412 (2012).

35. Chen, M. et al. GmDREB2, a soybean DRE-binding transcription factor, conferred drought and high-salt tolerance in transgenic plants. Biochem. Biophys. Res. Commun. 353, 299-305 (2007).

36. Yang, Z., Tian, L., Latoszek-Green, M., Brown, D. \& Wu, K. Arabidopsis ERF4 is a transcriptional repressor capable of modulating ethylene and abscisic acid responses. Plant Mol. Biol. 58, 585-596 (2005).

37. Hu, Y., Poh, H. M. \& Chua, N. H. The Arabidopsis ARGOS-LIKE gene regulates cell expansion during organ growth. Plant J. 47, 1-9 (2006).

38. Feng, G., Qin, Z., Yan, J., Zhang, X. \& Hu, Y. Arabidopsis ORGAN SIZE RELATED1 regulates organ growth and final organ size in orchestration with ARGOS and ARL. N. Phytol. 191, 635-646 (2011).

39. Chen, K. et al. Abscisic acid dynamics, signaling and functions in plants. J. Integr. Plant Biol. 62, 25-54 (2019). 
40. Jensen, M. K. et al. ATAF1 transcription factor directly regulates abscisic acid biosynthetic gene NCED3 in Arabidopsis thaliana. FEBS Open Biol. 3, 321-327 (2013).

41. Sato, $\mathrm{H}$. et al. Arabidopsis thaliana NGATHA1 transcription factor induces ABA biosynthesis by activating NCED3 gene during dehydration stress. Proc. Natl Acad. Sci. USA 115, E11178-E11187 (2018).

42. luchi, S. et al. Regulation of drought tolerance by gene manipulation of 9-cisepoxycarotenoid dioxygenase, a key enzyme in abscisic acid biosynthesis in Arabidopsis. Plant J. 27, 325-333 (2001).

43. Xu, P. \& Cai, W. Functional characterization of the BnNCED3 gene in Brassica napus. Plant Sci. 256, 16-24 (2017).

44. Davière, J.-M. \& Achard, P. Gibberellin signaling in plants. Dev. Cell $\mathbf{1 4 0}$ 1147-1151 (2013).

45. Khush, G. S. Green revolution: the way forward. Nat. Rev. Genet. 2, 815-822 (2001).

46. Achard, P. \& Genschik, P. Releasing the brakes of plant growth: how GAs shutdown DELLA proteins. J. Exp. Bot. 60, 1085-1092 (2009).

47. Peng, J. et al. 'Green revolution'genes encode mutant gibberellin response modulators. Nature 400, 256-261 (1999).

48. Achard, P. et al. Gibberellin signaling controls cell proliferation rate in Arabidopsis. Curr. Biol. 19, 1188-1193 (2009).

49. Dill, A., Thomas, S. G., Hu, J., Steber, C. M. \& Sun, T.-p The Arabidopsis F-box protein SLEEPY1 targets gibberellin signaling repressors for gibberellininduced degradation. Plant Cell 16, 1392-1405 (2004)

50. Muangprom, A. \& Osborn, T. Characterization of a dwarf gene in Brassica rapa, including the identification of a candidate gene. Theor. Appl. Genet. 108 1378-1384 (2004).

51. Muangprom, A., Thomas, S. G., Sun, T.-p \& Osborn, T. C. A novel dwarfing mutation in a green revolution gene from Brassica rapa. Plant Physiol. 137 931-938 (2005).

52. Wang, Y., Huang, S., Liu, Z., Tang, X. \& Feng, H. Changes in endogenous phytohormones regulated by microRNA-target mRNAs contribute to the development of Dwarf Autotetraploid Chinese Cabbage (Brassica rapa L. ssp. pekinensis). Mol. Genet. Genomics 293, 1535-1546 (2018).

53. Martín-Trillo, M. \& Cubas, P. TCP genes: a family snapshot ten years later Trends Plant Sci. 15, 31-39 (2010).

54. Du, J. et al. Genome-wide identification and characterization of BrrTCP transcription factors in Brassica rapa ssp. rapa. Front. Plant Sci. 8, 1588 (2017).

55. Kim, J. H. \& Tsukaya, H. Regulation of plant growth and development by the GROWTH-REGULATING FACTOR and GRF-INTERACTING FACTOR duo. J. Exp. Bot. 66, 6093-6107 (2015).

56. Rodriguez, R. E., Ercoli, M. F., Debernardi, J. M. \& Palatnik, J. F. in Plant Transcription Factors (ed. Gonzalez, D.) 269-280 (Elsevier, 2016)

57. Kim, J. H., Choi, D. \& Kende, H. The AtGRF family of putative transcription factors is involved in leaf and cotyledon growth in Arabidopsis. Plant J. $\mathbf{3 6}$ 94-104 (2003).

58. Kim, J. H. \& Lee, B. H. Growth-regulating FACTOR4 ofArabidopsis thaliana is required for development of leaves, cotyledons, and shoot apical meristem. J. Plant Biol. 49, 463-468 (2006).

59. Horiguchi, G., Kim, G. T. \& Tsukaya, H. The transcription factor AtGRF5 and the transcription coactivator AN3 regulate cell proliferation in leaf primordia of Arabidopsis thaliana. Plant J. 43, 68-78 (2005).

60. Wang, F. et al. Genome-wide identification and analysis of the growthregulating factor family in Chinese cabbage (Brassica rapa L. ssp. pekinensis). BMC Genomics 15, 807 (2014).

61. Hong, J. K. et al. Overexpression of Brassica rapa GROWTH-REGULATING FACTOR genes in Arabidopsis thaliana increases organ growth by enhancing cell proliferation. J. Plant Biotechnol. 44, 271-286 (2017).

62. Rodriguez, R. E. et al. Control of cell proliferation in Arabidopsis thaliana by microRNA miR396. Dev. Cell 137, 103-112 (2010).

63. Wang, L. et al. miR396-targeted AtGRF transcription factors are required for coordination of cell division and differentiation during leaf development in Arabidopsis. J. Exp. Bot. 62, 761-773 (2011)

64. Swaminathan, K., Peterson, K. \& Jack, T. The plant B3 superfamily. Trends Plant Sci. 13, 647-655 (2008).

65. Alvarez, J. P. et al. Endogenous and synthetic microRNAs stimulate simultaneous, efficient, and localized regulation of multiple targets in diverse species. Plant Cell 18, 1134-1151 (2006).

66. Alvarez, J. P., Goldshmidt, A., Efroni, I., Bowman, J. L. \& Eshed, Y. The NGATHA distal organ development genes are essential for style specification in Arabidopsis. Plant Cell 21, 1373-1393 (2009).
67. Kwon, S. H. et al. Overexpression of a Brassica rapa NGATHA gene in Arabidopsis thaliana negatively affects cell proliferation during lateral organ and root growth. Plant Cell Physiol. 50, 2162-2173 (2009).

68. Zhang, Y. et al. Transcription factors SOD7/NGAL2 and DPA4/NGAL3 act redundantly to regulate seed size by directly repressing $\mathrm{KLU}$ expression in Arabidopsis thaliana. Plant Cell 27, 620-632 (2015).

69. Bartel, D. P. MicroRNAs: genomics, biogenesis, mechanism, and function. Cell 116, 281-297 (2004)

70. Mallory, A. C., Elmayan, T. \& Vaucheret, H. MicroRNA maturation and actionthe expanding roles of ARGONAUTEs. Curr. Opin. Plant Biol. 11, 560-566 (2008)

71. Palatnik, J. F. et al. Control of leaf morphogenesis by microRNAs. Nature $\mathbf{4 2 5}$ 257-263 (2003).

72. Schommer, C. et al. Control of jasmonate biosynthesis and senescence by miR319 targets. PLoS Biol. 6, e230 (2008).

73. Mao, Y. et al. MicroRNA319a-targeted Brassica rapa ssp. pekinensis TCP genes modulate head shape in Chinese cabbage by differential cell division arrest in leaf regions. Plant Physiol. 164, 710-720 (2014)

74. Song, J. B., Huang, S. Q., Dalmay, T. \& Yang, Z. M. Regulation of leaf morphology by microRNA394 and its target leaf curling responsiveness. Plant Cell Physiol. 53, 1283-1294 (2012).

75. Knauer, S. et al. A protodermal miR394 signal defines a region of stem cell competence in the Arabidopsis shoot meristem. Dev. Cell 24, 125-132 (2013)

76. Song, J. B. et al. Altered fruit and seed development of transgenic rapeseed (Brassica napus) over-expressing microRNA394. PLOS ONE 10, e0125427 (2015)

77. Li, Y., Zheng, L., Corke, F., Smith, C. \& Bevan, M. W. Control of final seed and organ size by the DA1 gene family in Arabidopsis thaliana. Genes Dev. 22 1331-1336 (2008).

78. Dong, $\mathrm{H}$. et al. Ubiquitylation activates a peptidase that promotes cleavage and destabilization of its activating E3 ligases and diverse growth regulatory proteins to limit cell proliferation in Arabidopsis. Genes Dev. 31, 197-208 (2017)

79. Vanhaeren, $\mathrm{H}$. et al. Forever young: the role of ubiquitin receptor DA1 and E3 ligase BIG BROTHER in controlling leaf growth and development. Plant Physiol. 173, 1269-1282 (2017).

80. Wang, J. L. et al. Down-regulation of BnDA1, whose gene locus is associated with the seeds weight, improves the seeds weight and organ size in Brassica napus. Plant Biotechnol. J. 15, 1024-1033 (2017).

81. Peng, $Y$. et al. The ubiquitin receptors DA1, DAR1, and DAR2 redundantly regulate endoreduplication by modulating the stability of TCP14/15 in Arabidopsis. Plant Cell 27, 649-662 (2015).

82. Macknight, R. et al. FCA, a gene controlling flowering time in Arabidopsis, encodes a protein containing RNA-binding domains. Cell $\mathbf{8 9}, 737-745$ (1997).

83. Hornyik, C., Terzi, L. C. \& Simpson, G. G. The spen family protein FPA controls alternative cleavage and polyadenylation of RNA. Dev. Cell 18, 203-213 (2010)

84. Liu, F., Marquardt, S., Lister, C., Swiezewski, S. \& Dean, C. Targeted 3' processing of antisense transcripts triggers Arabidopsis FLC chromatin silencing Science 327, 94-97 (2010).

85. Sun, F. et al. A conserved RNA recognition motif (RRM) domain of Brassica napus FCA improves cotton fiber quality and yield by regulating cell size. Mol. Breed. 30, 93-101 (2012).

86. Hong, F. et al. Overexpression of the r FCA RNA recognition motif affects morphologies modifications in rice (Oryza sativa L.). Biosci. Rep. 27, 225-234 (2007).

87. Attia, K. et al. Transformation and functional expression of the rFCA-RRM2 gene in rice. J. Integr. Plant Biol. 47, 823-830 (2005).

88. Qi, W. et al. Over-expression of a conserved RNA-binding motif (RRM) domain (csRRM2) improves components of Brassica napus yield by regulating cell size. Plant Breed. 131, 614-619 (2012)

89. Li, J., Li, G., Wang, H. \& Deng, X. W. Phytochrome signaling mechanisms. Arabidopsis Book 9, e0148 (2011).

90. Childs, K L et al. The sorghum photoperiod sensitivity gene, Ma3, encodes a phytochrome B. Plant Physiol. 113, 611-619 (1997).

91. Reed, J. W., Nagpal, P., Poole, D. S., Furuya, M. \& Chory, J. Mutations in the gene for the red/far-red light receptor phytochrome B alter cell elongation and physiological responses throughout Arabidopsis development. Plant Cell 5, 147-157 (1993). 
92. Sheehan, M. J., Kennedy, L. M., Costich, D. E. \& Brutnell, T. P. Subfunctionalization of PhyB1 and PhyB2 in the control of seedling and mature plant traits in maize. Plant J. 49, 338-353 (2007).

93. Wagner, D., Tepperman, J. M. \& Quail, P. H. Overexpression of phytochrome B induces a short hypocotyl phenotype in transgenic Arabidopsis. Plant Cell 3, 1275-1288 (1991).

94. Song, M.-F. et al. Ectopic expression of a phytochrome B gene from Chinese cabbage (Brassica rapa L. ssp. pekinensis) in Arabidopsis thaliana promotes seedling de-etiolation, dwarfing in mature plants, and delayed flowering. Plant Mol. Biol. 87, 633-643 (2015).

95. Pérez-Pérez, J. M., Serrano-Cartagena, J. \& Micol, J. L. Genetic analysis of natural variations in the architecture of Arabidopsis thaliana vegetative leaves. Genetics 162, 893-915 (2002).

96. Frary, A., Fritz, L. A. \& Tanksley, S. D. A comparative study of the genetic bases of natural variation in tomato leaf, sepal, and petal morphology. Theor. Appl. Genet. 109, 523-533 (2004).
97. Chitwood, D. H. et al. A modern ampelography: a genetic basis for leaf shape and venation patterning in grape. Plant Physiol. 164, 259-272 (2014).

98. Ku, L. et al. Genetic analysis of leaf morphology underlying the plant density response by QTL mapping in maize (Zea mays L.). Mol. Breed. 36, 63 (2016).

99. Lan, T.-H. \& Paterson, A. Comparative mapping of QTLs determining the plant size of Brassica oleracea. Theor. Appl. Genet. 103, 383-397 (2001)

100. Lou, P. et al. Quantitative trait loci for flowering time and morphological traits in multiple populations of Brassica rapa. J. Exp. Bot. 58, 4005-4016 (2007)

101. Choi, S. R. et al. Integrated analysis of leaf morphological and color traits in different populations of Chinese cabbage (Brassica rapa ssp. pekinensis). Theor. Appl. Genet. 130, 1617-1634 (2017).

102. Xiao, D. et al. Genetic dissection of leaf development in Brassica rapa using a genetical genomics approach. Plant Physiol. 164, 1309-1325 (2014).

103. Jian, H. et al. Screening of candidate leaf morphology genes by integration of QTL mapping and RNA sequencing technologies in oilseed rape (Brassica napus L.). PLoS ONE 12, e0169641 (2017). 